\title{
Anastomosis terminal funcional con sutura mecánica en cirugía intestinal. Resultados precoces de una serie prospectiva*
}

\author{
Drs. ALEJANDRO BARRERA E. ${ }^{1}$, GUILLERMO BANNURA C. ${ }^{1}$, FELIPE ILLANES F. ${ }^{1}$, CARLOS MELO L. ${ }^{1}$ \\ 1 Servicio y Departamento de Cirugía, Hospital Clínico San Borja Arriarán, Campus Centro, Facultad de Medicina, \\ Universidad de Chile \\ Santiago, Chile.
}

\begin{abstract}
Experience with mechanical anastomoses in bowel surgery

Background: The use of mechanical anastomoses reduce the length of gastrointestinal surgical procedures. Aim: To report the experience with mechanical functional terminal anastomoses in bowel surgery. Material and Methods: Prospective non randomized registry of all mechanical anastomoses carried out in a surgical service. Results: A total of 327 patients (193 women) were analyzed. Two hundred and one anastomoses were ileo-colonic and 126 were entero-enteral. Seventeen percent of patients experienced some complication. Fourteen required a new operation due to peritonitis or deep infection of the surgical site. The global rate of anastomotic dehiscence was 3.1\%. No differences in the rate of complications between surgeons with experience and trainees were observed. Conclusions: Mechanical anastomoses in bowel surgery are safe and require a short learning period to be used.
\end{abstract}

Key words: Mechanical suture, anastomoses, dehiscence.

\section{Resumen}

Se presenta nuestra experiencia con la anastomosis terminal funcional con sutura mecánica en cirugía intestinal. La serie corresponde a 327 pacientes, un 59\% de género femenino. Del total de anastomosis 201 fueron íleo colónicas y 126 entero enterales. El instrumento de sutura más utilizado fue de 75 u $80 \mathrm{~mm}$ de longitud y no se registran incidentes durante la confección de la anastomosis. La morbilidad específica se encuentra en el $17 \%$ de la serie, sin diferencias entre los dos tipos de anastomosis. Catorce paciente fueron reoperados por peritonitis o infección profunda del sitio quirúrgico La tasa global de dehiscencia anastomótica fue de un 3,1\% ( 5 y 4 casos respectivamente) sin alcanzar significación estadística. Al comparar si la anastomosis fue realizada por un cirujano con experiencia o en formación, tampoco se encontraron diferencias estadísticamente significativas, por lo que nos parece una técnica segura y con una corta curva de aprendizaje.

Palabras clave: Anastomosis terminal funcional, sutura mecánica.

*Recibido el 22 de agosto de 2011 y aceptado para publicación el 15 de septiembre de 2011.

Correspondencia: Dr. Alejandro Barrera E. Apoquindo 8160 Dpto. 31, Santiago, Chile. abarrera@med.uchile.cl 


\section{Introducción}

Con la utilización de aparatos de sutura mecánica en cirugía colorrectal se han logrado anastomosis seguras y con un menor tiempo operatorio ${ }^{1-3}$. Sus efectos más significativos se han alcanzado en la cirugía del recto bajo, permitiendo anastomosis más seguras y funcionales, donde es casi imposible una sutura manual ${ }^{4,5}$.

La técnica de anastomosis intestinal terminal funcional introducida por Ravitch y Steichen ${ }^{6,7}$, ha permitido construir una anastomosis con escasa manipulación de los cabos a anastomosar, lo que permite mantener el aporte sanguíneo en ambos bordes además de obtener una anastomosis de amplio lumen, lo que teóricamente se traduce en una recuperación más rápida del tránsito intestinal y una menor estada hospitalaria ${ }^{8,9}$. A nivel nacional esta técnica ha sido utilizada para la anastomosis posterior a una colectomía derecha ${ }^{10} \mathrm{y}$ para la reconstitución del tránsito luego de una ileostomía en asa $^{11}$.

El objetivo de este estudio es determinar el espectro clínico de los pacientes en que se realizó una anastomosis terminal funcional, evaluar la morbilidad específica y conocer los resultados inmediatos de esta cirugía en una serie prospectiva, consecutiva, no aleatoria.

\section{Material y Método}

Desde el año 1998 se inició en nuestro Servicio un registro prospectivo, consecutivo y no aleatorio sobre el uso de suturas mecánicas en cirugía colorrectal. De este registro se seleccionan aquellos pacientes en los que se realizó una anastomosis mecánica terminal funcional luego de una resección de intestino grueso o delgado o para una reconstitución del tránsito. Se registran sus datos epidemiológicos, patología agregada, tipo de anastomosis (entero entero anastomosis (EEA) o entero colo anastomosis (ECA)), tiempo operatorio, incidentes quirúrgicos, confección de la anastomosis por cirujano o médico en formación y resultados precoces de la cirugía, considerando el tiempo de realimentación, duración del íleo pos operatorio, morbilidad global y especifica. Se definió como mortalidad operatoria la intra hospitalaria, incluyendo en esta aquellos pacientes que reingresaron por alguna complicación médica o quirúrgica luego del alta, independiente del tiempo transcurrido de la cirugía.

Los datos fueron recolectados en una planilla Excel (Microsoft@) y para el análisis estadístico se utilizó el paquete Stata 8.0, considerando significativo un valor de $\mathrm{p}<0,05$.

\section{Resultados}

Durante el período señalado se operaron 327 pacientes en los que se realizó una anastomosis mecánica terminal funcional. Se encontró un predominio del género femenino con 193 pacientes (59\%). Doscientos doce (64\%) presentaban una o más patologías agregadas, entre las que predominaron la hipertensión arterial y diabetes mellitus. Del total de anastomosis, 201 (61\%) fueron entero colo anastomosis y 126 (39\%) entero entero anastomosis. La mediana de edad de la serie fue de 66 años, con extremos de 15 y 88. Entre las ECA predominó como diagnóstico pre operatorio el cáncer de colon derecho ( $90 \%$ de los casos) y en la EEA el cierre de una ileostomía en asa (78\%).

En 291 casos (89\%) se utilizó un aparato de sutura lineal cortante de 80 ó $75 \mathrm{~mm}$ con una carga para completar la sutura. (Tabla 1) En todos los casos se utilizó refuerzo de la sutura mecánica con un punto de poliglactina 3-0 en el vértice de la anastomosis latero lateral y una sutura continua del mismo material a lo largo de la segunda sutura lineal cortante a modo de hemostasia.

En 164 pacientes se utilizó anestesia regional (espinal o peridural) y en los restantes anestesia general. Existe una diferencia altamente significativa al comparar ambos tipos de anastomosis, ya que en las ECA se utilizó preferentemente anestesia general y en la EEA anestesia regional (Tabla 2).

El tiempo operatorio promedio fue de 91 minutos, 68 minutos para la EEA y 127 minutos para la ECA $(p=0,001)$. No se encontraron diferencias significativas en el tiempo que demora la eliminación de gases por ano, duración del íleo pos operatorio ni en el tiempo de realimentación oral.

Tabla 1. Tipos de sutura

\begin{tabular}{|c|c|}
\hline Tipo de sutura & n \\
\hline $75-80 \mathrm{~mm}$ & 252 \\
\hline $55-60 \mathrm{~mm}$ & 29 \\
\hline $100 \mathrm{~mm}$ & 1 \\
\hline Total & 282 \\
\hline
\end{tabular}

Tabla 2. Anestesia

\begin{tabular}{|lccc|}
\hline & $\begin{array}{c}\text { Anestesia } \\
\text { regional }\end{array}$ & $\begin{array}{c}\text { Anestesia } \\
\text { general }\end{array}$ & $\mathbf{p}$ \\
\hline EEA & 93 & 12 & $<0,01$ \\
ECA & 49 & 128 & $<0,01$ \\
\hline
\end{tabular}


Tabla 3. Morbilidad específica

\begin{tabular}{|lrcc|}
\hline & EEA & ECA & p \\
\hline Infección sitio quirúrgico & 11 & 14 & ns \\
\hline Íleo post operatorio & 6 & 9 & ns \\
Dehiscencia anastomótica & 4 & 5 & ns \\
Total & 21 & 28 & ns \\
\hline
\end{tabular}

Con respecto a la morbilidad específica, esta se registró en 49 pacientes (17\% de la serie global). (Tabla 3) Catorce pacientes fueron reintervenidos por infección profunda del sitio quirúrgico o peritonitis $(4,9 \%)$ sin encontrar diferencias significativas en ambos grupos. La tasa global de dehiscencia fue de un 3,1\%. Se presentaron 5 casos en el grupo de ECA y 4 en el de EEA. El análisis estadístico no mostró diferencias significativas ( $p=0,87$ ).

La estada hospitalaria promedio fue de 9 días, lo que se eleva a 15 en los pacientes complicados. Tampoco se encontraron diferencias significativas al comparar ambos grupos.

Se registraron dos casos de mortalidad operatoria, ambos en el grupo de ECA, con lo que la tasa para este grupo alcanza al $1 \%$ y para la serie global a un $0,6 \%$.

Se comparó también la experiencia del cirujano que confeccionaba la anastomosis, según si había completado su formación quirúrgica. No se encontraron diferencias significativas en ninguno de los parámetros evaluados entre los cirujanos que se encontraban en período de formación y en aquellos que ya lo habían terminado.

\section{Discusión}

Los instrumentos de sutura mecánica de tipo lineal cortante están disponibles para su uso desde mediados de los 60. Inicialmente fueron utilizados en cirugía gástrica para la confección de anastomosis latero laterales en reconstrucciones tipo Billroth 2 y en cirugía de colon para la reconstitución del tránsito posterior a una colostomía en doble caño ${ }^{6}$. La técnica terminal funcional original incluía la confección de la anastomosis latero lateral con un aparato lineal cortante y luego el cierre de los cabos con dos disparos de una sutura lineal de 30 ó 55 $\mathrm{mm}^{12}$. Con posterioridad esta técnica fue modificada, reemplazando los disparos de sutura lineal con una carga de instrumento lineal cortante, lo que hace más económico el procedimiento, con un resultado semejante ${ }^{8,13}$.

Mucho se discutió en los inicios de esta técnica acerca de la posible morbilidad relacionada con la aposición mucosa a mucosa del segundo disparo de sutura mecánica, lo que podría derivar en una mayor proporción de dehiscencias. Distintos trabajos demostraron que no existen alteraciones de la cicatrización y que la fuerza tensil en la línea de sutura es comparable a una anastomosis con aposición serosa a serosa ${ }^{14,15}$. La única diferencia se encontró en modelos experimentales, en que este tipo de anastomosis era menos resistente a los incrementos de presión, sin embargo, estos hallazgos no han tenido traducción clínica ${ }^{14}$. La técnica utilizada en la actualidad es una mezcla de aposición serosa a serosa en la anastomosis latero lateral y mucosa a mucosa en el cierre de los cabos, con lo que se obtienen los beneficios de ambos métodos sin agregar morbilidad o dificultad en su confección.

Entre las ventajas descritas para esta técnica están la mantención del aporte sanguíneo en ambos bordes, la rapidez y escaso traumatismo en la confección y el amplio diámetro de la anastomosis. Todos estos beneficios podrían traducirse en un menor tiempo operatorio, menor edema y por lo tanto, una más rápida recuperación del tránsito intestinal y una menor frecuencia de dehiscencia ${ }^{8,16}$. La literatura refiere que en los distintos tipos de cirugía en que esta anastomosis se utiliza, observamos alguna de estas ventajas. En el cierre de una ileostomía en asa se ha observado una disminución significativa del tiempo operatorio, sin beneficios en la disminución de una dehiscencia ni en las complicaciones obstructivas $^{16,17}$. Probablemente los mayores beneficios de la técnica se observan en las resecciones ileocolónicas por enfermedad de Crohn. En este caso se ha demostrado una menor tasa de dehiscencia y menor recurrencia de estenosis al compararla con la sutura manual $^{18}$.

En relación al cáncer de colon, la principal indicación de esta técnica está en el restablecimiento del tránsito intestinal luego de una resección de colon derecho ${ }^{5,10,19}$. Esta anastomosis puede ser realizada antes de la resección colónica (técnica cerrada) o una vez realizada (técnica abierta). Creemos que la técnica cerrada al exponer el lumen intestinal por menor período de tiempo resulta en una menor contaminación del campo operatorio y teóricamente menor incidencia de complicaciones sépticas. Sin embargo, este beneficio teórico no ha sido demostrado por la literatura. En la resección laparoscópica del cáncer de colon puede ser realizada en forma intra corpórea, técnica que no utilizamos, ya que la extracción de la pieza operatoria requiere una mini laparotomía que permite la realización de la técnica fuera del campo operatorio con comodidad y seguridad.

Una revisión sistemática publicada en forma 
reciente encuentra beneficios en una menor tasa de dehiscencia tanto en las anastomosis ileocolónicas por cáncer como en enfermedad de Crohn, sin diferencias significativas en las tasas de estenosis, sangrado anastomótico, reoperaciones, mortalidad ni complicaciones sépticas ${ }^{19}$.

Un factor no evaluado por la literatura es la curva de aprendizaje necesaria para la confección de una anastomosis intestinal. En esta serie se comparó aquellas anastomosis realizadas por cirujanos con experiencia con las realizadas por residentes de cirugía, asistidos por un cirujano con formación completa y no encontramos diferencias en ninguno de los parámetros analizados. Probablemente este hecho esté dado por la estandarización de la sutura dado por el instrumento mecánico, la facilidad de su uso y por ende una curva de aprendizaje más corta para lograr una utilización correcta y con buenos resultados iniciales.

En suma, se presenta una serie prospectiva, consecutiva, no aleatoria de 327 pacientes con una anastomosis terminal funcional, la morbilidad específica alcanza un 17\%, la tasa de dehiscencia a un $3,1 \%$ y la mortalidad operatoria de un $0,6 \%$.

\section{Referencias}

1. Hansen O, Schwenk W, Hucke HP, Stock W. Colorectal stapled anastomoses. Experiences and results. Dis Colon Rectum 1996;39:30-6.

2. Morán BJ. Stapling instruments for intestinal anastomosis in colorectal surgery. Br J Surg. 1996;83:902-9.

3. Bannura G, Melo C, Contreras J, Barrera A, Villaman J, Lee K. Suturas mecánicas en cirugía colorrectal: análisis de 225 pacientes consecutivos. Rev Chil Cir. 2002;54:350-7.

4. Law WI, Chu K-W, Chan C-W. Risk factors for anastomosis leakage after low anterior resection with total mesorectal excision. Am J Surg. 2000;79;1:92-6.

5. MacRae HM, McLeod RS. Handsewn vs stapler anastomoses in colon and rectal surgery. A meta-analysis. Dis Colon Rectum 1998;41:180-9.

6. Steichen FM. The use of stapler in anatomical side- to-side and functional end-to-end enteroanastomoses. Surgery 1968;64:648-53.

7. Ravitch MM, Rivarola A. Enteroanastomosis with an automatic instrument. Surgery 1966;59:270-7.

8. Kestenberg A, Becker J. A new technique of loop ileostomy closure after endorectal ileoanal anastomosis. Surgery 1985;98:109-11.

9. Meagher AP, Wolff BG. Right hemicolectomy with a linear cutting stapler. Dis Colon Rectum 1994;37:10435.

10. Albarrán V, Alamo M, Blake P, Hermansen C, Bardavid C, Guzmán L y cols. Hemicolectomía derecha. Técnica semicerrada. Rev Chil Cir. 1998;50:642-5.

11. Barrera A. Cierre ileostomía en asa con sutura mecánica. Análisis prospectivo de 25 pacientes consecutivos. Rev Chil Cir. 2003;55:573-9.

12. Ravitch M, Lane R, Cornell W, Rivarola A, McEnany T. Closure of duodenal and gastric stump with wire staples, experimental and clinic studies. Ann Surg. 1966;59:573-9.

13. Chassin JL, Rifkind KM, Turner JW. Errors and pitfalls in stapling gastrointestinal anastomoses. Surg Clin N A. 1984;64:441-59.

14. Getzen LC. Clinical use of everted intestinal anastomosis. Surg Ginecol and Obst. 1966;123:1027-36.

15. Ravitch M, Canalis F, Wainshelbaum A, McCormick J. Studies in intestinal healing III. Observations on everting intestinal anastomoses. Ann Surg. 1967;166:67080.

16. Hasegawa H, Radley S, Morton DG, Keiglhey MRD. Stappled vs sutured closure of loop ileostomy. A randomized controled trial. Ann Surg. 2000;231:202-4.

17. Hull T, Kobe I, Fazio V. Comparison of handsewn and stapled ileostomy closure. Dis Colon Rectum 1995;39:1086-9.

18. Resegotti A, Astegiano M, Farina E, Ciggone G, Avagnina G, Giustteto A, et al. Side to side stapled anastomosis strongly reduces anastomotic leak rates in Crohn's diseases surgery. Dis Colon Rectum 2005;48:464-8.

19. Choy PYG, Bisset YP, Docherty JG, Parry BR, Merrie A. Stapled versus handsewn methods for ileocolonic anastomoses. (Review) Cochrane Database of systematic review 2007, Issue 3 art No.: CD004320. DOI: 10.1002/14651858.CD004320.pub2. 